\title{
Germanica
}

\section{Ulysse de Danemark et la dialectique du désir autour de Henrik Nordbrandt et de Johannes $\mathrm{V}$. Jensen}

Ödüsseus oder die Dialektik der Sehnsucht

Karl Ejby Poulsen

\section{(2) OpenEdition}

\section{Journals}

Édition électronique

URL : http://journals.openedition.org/germanica/2196

DOI : 10.4000/germanica.2196

ISSN : 2107-0784

Éditeur

Université de Lille

Édition imprimée

Date de publication : 31 décembre 2001

Pagination : 101-114

ISBN : 9782913857063

ISSN : 0984-2632

\section{Référence électronique}

Karl Ejby Poulsen, «Ulysse de Danemark et la dialectique du désir autour de Henrik Nordbrandt et de Johannes V. Jensen », Germanica [En ligne], 29 | 2001, mis en ligne le 20 septembre 2013, consulté le 06 octobre 2020. URL : http://journals.openedition.org/germanica/2196 ; DOI : https://doi.org/

10.4000/germanica.2196

Ce document a été généré automatiquement le 6 octobre 2020.

(c) Tous droits réservés 


\section{Ulysse de Danemark et la dialectique du désir autour de Henrik Nordbrandt et de Johannes V. Jensen}

Ödüsseus oder die Dialektik der Sehnsucht

\section{Karl Ejby Poulsen}

Ô toi, promeneur, connais-tu le mot de passe pour entrer en toimême.

(Adonis)

l'attente fut ta limite ton désir hors du monde, sa racine secrète

(Paul la Cour)

Seul un mort entrera doucement en lui-même au travers de ces murs blancs

(Henrik Nordbrandt)

Henrik Nordbrandt (né en 1945) ayant exprimé son admiration, sinon pour le poète Johannes V. Jensen, au moins pour l'auteur de La Chute du roi, la comparaison des deux écrivains paraît de ce point de vue légitime. L'on pourrait appeler dialectique du désir, ce qui les unit. Et le voyage. Celui de Johannes V. Jensen (1873-1950) dura un an; en 1902 il fit le tour du monde. Celui de Henrik Nordbrandt dure encore - concrètement, mais aussi dans ce sens que le voyage, chez Nordbrandt, est un modus vivendi existentiel, une idée poétique et la métaphore centrale de l'existence.

2 Le voyage de Johannes $\mathrm{V}$. Jensen est réuni dans six poèmes sous le titre Le voyage autour de la terre. Le point de départ est un manque, mal défini et surtout exprimé par la négative, par le malaise d'être au Danemark : 
Grisaille à Copenhague !

Rhumatismes et virulence pour les siècles des siècles!

Le manque produit un désir qui l'empêche de s'installer dans ce même Danemark. Il part donc pour trouver le lieu qui pourra satisfaire ce désir. Mais où qu'il soit, le désir se redouble et l'empêche de s'installer. A la gare de Memphis est une belle démonstration de cette dialectique du désir : pour satisfaire le désir le lieu doit avoir les exactes mesures du désir - que seul le désir peut lui donner. On assiste donc à un formidable gonflement du réel par l'imagination qui dure le temps que dure l'énergie imaginaire :

Regarde comment l'immense fleuve jouit de sa courbe !

Comment il déborde royalement dans sa courbe et fait pirouetter des armadas

d'arbres et de débris de bois flottants dans ses rapides!

Regarde comment il conduit un énorme bateau à roues

dans ses bras diluviens,

comme un danseur qui est maître du parquet !

Regarde les pointes immergées - Oh le calme primitif et grandiose

de ce paysage de forêts noyées !

$\mathrm{Ne}$ vois-tu pas comment les eaux matinales du flot

revêtent à perte de vue la lumière simple du jour

et marchent sainement sous les nuages pleins !1

Puis c'est de nouveau la grisaille et la pluie,

Et nous continuons le voyage

à travers les forêts inondées

sous les cataractes béantes de la pluie ${ }^{2}$.

Le but du voyage est de consumer ce désir afin de pouvoir enfin se sédentariser. Johannes V. Jensen n'y arrive pas et doit partager, à sa façon, le destin de Christophe Colomb à qui il a consacré un de ses textes les plus pénétrants à ce sujet. Connaissant le lieu où le soleil se lève, Colomb part à la recherche de l'endroit qu'il éclaire quand il est couché. Le but de son désir est donc, en d'autres termes, l'absolu. Mais quand l'Amérique est en vue et que les marins pleurent de joie tout en nommant Colomb libérateur du monde, lui se tait:

Car lorsqu'il trouva l'île salvatrice

son rêve périt ${ }^{3}$

Le désir perd son objet, tout objet, se dédouble en forlæengsel (désir-désir), terme danois qui indique un état qui n'est que du désir, comme « forstenet » (pétrifié) indique un état où il n'y a que de la pierre et "fortvivlet " (désespéré) un état où il n'y a que du désespoir. Colomb se confond avec son désir-désir, voué à naviguer éternellement sous forme de spectre : esprit/désir sans corps. Une sorte d'état de mort au monde. C'est ce spectre qui hantera partout dans l'œuvre de Johannes V. Jensen : le rêve de l'absolu et qu'il satisfera en proposant sa propre version du darwinisme qui, à cet égard, n'est autre qu'un investissement de ce désir dans le temps, au lieu d'un investissement dans l'espace, et qu'il matérialisera dans les six romans qui constituent Den lange Rejse (Le long voyage), voyage en arrière, à la recherche des forêts perdues d'où vient l'homme du Nord. Mais le résultat est identique à ce qui se passe dans À la gare de Memphis, à la différence que l'énergie $e^{4}$ de l'imagination dure plus longtemps, nourrie par une espèce de concentration d'énergie que l'on voit poindre dans La quotidienneté, le dernier poème du tour du monde :

Écoute! Des tirs au loin,

comme des éboulements souterrains, derrière les collines moissonnées. 
L'été referme ses cercueils.

Nous ne sommes donc plus jeunes.

Mais est-ce que l'espoir ne nous a pas suffisamment confondus?

Voici venu notre riche temps de la mort, mon frère.

Entrons donc impavides dans la quotidienneté,

nous, qui n'avons rien à perdre.

Faisons-en un jeu mordant,

une rude séance de prières

pour les célèbres deux millions d'habitants

du royaume du Danemark ! ${ }^{5}$

Décidément : le désir insatiable rend la volupté impossible.

Le destin de Johannes V. Jensen - qu'il partageait en quelque sorte avec Karen Blixen fut celui, paradoxal, que tout en souhaitant ardemment sortir de la littérature, de l'écriture, il s'y enfonça de plus en plus. Son destin fut en quelque sorte d'être celui qui ne veut pas être celui qu'il est et qui ne peut pas être celui qu'il veut être.

Chez Henrik Nordbrandt, on trouve une formule identique :

Je continue de rêver du lieu

à la description duquel cette phrase

renoncera aussi ${ }^{6}$

10 Le poème, la description du lieu rêvé, est le signe douloureux que ce lieu n'est pas encore atteint. Il naît précisément de la distance entre rêve et lieu réel. Au fur et à mesure que le Moi-voyageur chez Nordbrandt s'approche de ce qu'il croit être le but du voyage, il voit qu'il s'est trompé ; le but s'efface et le Moi devient personne :

Je suis ce personne qu'Ithaque a fait de moi

L'Ithaque qui se reflète dans la mer

L'Ithaque que je croyais être mon désir

quand le désir avait encore une forme et pouvait être satisfait ${ }^{7}$

11 L'identité, c'est le voyage, non pas l'arrivée qui opère la perte de la forme du désir. Ce qu'il cherche est défini par ce qu'il quitte et qui a produit ce manque, ce vide que ce qu'il cherche doit combler. C'est donc logique que le but du voyage s'estompe au fur et à mesure qu'il s'approche de lui, car il n'était qu'une forme provisoire du désir. C'est pourquoi le voyage, chez Nordbrandt, prend la forme d'une série ininterrompue de départs et arrivées. Départ aussitôt arrivé, parfois même avant d'être arrivé, car

Où que nous partions nous arriverons toujours trop tard

pour trouver ce que nous sommes partis chercher

Et quelle que soit la ville où nous séjournons

ce sont les maisons auxquelles il est trop tard de retourner

les jardins où il est trop tard pour passer une nuit au clair de lune

et les femmes qu'il est trop tard d'aimer

qui nous hantent avec leur présence impalpable ${ }^{8}$.

12 Telle semble être la dialectique du voyage ou du désir chez Henrik Nordbrandt qui dans Bichare ${ }^{9}$ se définit comme celui qui arrive, encore et encore et comme celui qui part toujours. 
forment, avec certaines de leurs traditions poétiques, le décor de la thématique essentiellement nordbrandtienne - et, faut-il dire, moderne - celle de l'identité et de l'absence ou plutôt : présence de l'absence. Il dit ce que ces paysages lui apportent, dans le poème Débarquement, dans Opbrud og ankomster (Départs et arrivées). Après, dit le Moi poétique, tant de voyages en vain, après avoir décrit les choses qu'il a vues tellement de fois qu'elles ont cessé d'exister pour ne rester que sous la forme de mots, il revit tout à coup chaque mot comme une déclaration d'amour :

Et j'adore chaque mot parce qu'il me force à chanter

de la même manière que nous chantons les tempêtes sur la mer

parce qu'elles nous forcent à capituler devant elles et à chercher refuge

dans tant de ports inconnus, sur tant d'îles enchantées.

J'adore les villes où nous fûmes maltraités, pour leurs noms,

les olives noires, le pain et le mot pour vin dans sept langues.

J'adore les pays que nous ne vîmes jamais parce qu'il nous forcèrent à les inventer.

J'adore la terre brûlante parce qu'elle me force à danser

et mes masques usés parce qu'ils me forcent à rire.

J'adore ma mort fortuite parce qu'elle me force à vivre ${ }^{12}$.

C'est une expérience du même ordre que celle du poète suédois Gunnar Ekelöf :

Quand on est allé aussi loin que moi dans l'absurdité

chaque mot redevient intéressant ${ }^{13}$.

D'entrée de jeu, le Moi nordbrandtien définit sa situation existentielle comme étant celle d'être suspendu

[...] entre une existence qu'il est trop tard de vivre

et une existence qui est inacceptable ${ }^{14}$.

19 Le poème pose la question du bonheur et décrit le paysage du bonheur, c'est-à-dire le paysage où la question du bonheur devient superflue parce que la réponse devient inexistante. C'est un paysage où l'extérieur et l'intérieur, le dessus et le dessous, l'avant et l'après coïncident ; un paysage

\section{[...] dont les distances}

nous font pénétrer de plus en plus loin en nous-mêmes

au fur et à mesure que nous les dépasserons, et où nos vies deviennent concrètes

comme les rochers abrupts qui les conditionnent $[. . .]^{15}$.

La vie concrète, élémentaire, ici dans ce paysage est opposée à la vie abstraite dans les métropoles de la civilisation moderne. La poésie de Nordbrandt contient une critique au vitriol de la vie contemporaine, abstraite et destructrice parce que son action consiste à faire valoir l'abstraction dans la réalité ce qui, d'après Hegel, détruit la réalité en la rendant abstraite à son tour, sans consistance, sans poids, sans sensualité, 
sans identité, sans authenticité. Ce qui produit un manque qui produit à son tour une abstraction qui sera projetée sur le réel - ainsi de suite dans une spirale infernale, une constante production d'abstractions, de vides. Nordbrandt lui oppose une autre expérience :

C'était nous, mon amour, qui ensemble créâmes ce moment

en dehors de nous.

Avec le son

du brisant, les ombres des oliviers et le sel

qui toucha nos corps avec un connaissance

de belles grottes et des mains blanches d'hommes noyés

qui ne pouvaient pas nous atteindre,

Il appartient à un après-midi

que nous laissâmes demeurer après-midi en le quittant à temps ${ }^{16}$.

21 Parce qu'il n'a pas, comme son collègue ès voyages Johannes $V$. Jensen, cédé au désir d'exiger tout de cet instant, mais a eu le courage de le quitter, l'instant lui donne tout et devient réalité. On peut dire que Nordbrandt, ici, à l'étranger fait l'expérience décrite par Emmanuel Lévinas que paradoxalement, c'est en tant qu'alinéus - étranger - que l'homme n'est pas aliéné ${ }^{17}$. Car, se sentir étranger à l'étranger c'est paradoxalement aussi se sentir chez soi. Quand le domestique, le connu, devient étranger, il se produit un sentiment de malaise, de vide, d'absence qui est celui des premiers recueils de Henrik Nordbrandt ainsi que celui de la plupart de ses recueils qui ont le Danemark comme lieu d'inspiration ${ }^{18}$. Quand l'étranger est au contraire vécu comme domestique, il se produit un sentiment de bien-être qui est celui qui se dégage des recueils inspirés par la Méditerrané ${ }^{19}$. Le mot «bien-être » ne doit pas être pris à la lettre; j'entends par ce terme le fait que les sentiments corollaires aux étranges états d'âme qu'éprouvait autrefois le Moi sont absents de ces poèmes. Que les paysages étrangers semblent seulement accueillir leurs traces, les exprimer selon la formule symboliste : le paysage est un état d'âme - comme dans Crète où le paysage est devenu :

[...] un état d'âme

chez celui qui le parcourt : une schizophrénie

imposée de force par la chute des rivières et le bâillement

des ravins qu'aucun sommeil de marmotte ne peut refermer

et par la distance entre la neige et le pollen :

Une danse dont chaque pas t'éloigne

du Moi que tu pensais congénital :

Un départ qui t'embrasse à mort avec ton arrivée ${ }^{20}$.

Conscient et inconscient, rêve et conscience diurne, intérieur et extérieur se côtoient et s'approfondissent mutuellement. Il émane de ces poèmes un certain bonheur : celui de pouvoir simplement écrire, dire.

Le thème principal des poèmes méditerranéens est avant tout celui de l'identité. Ainsi le recueil Glas (Verre) commence par huit sonnets consacrés à ce sujet. Il est bien connu qu'on se déplace, voyage, pour se distancier, pour voir le lieu d'où l'on vient à distance. C'est le thème du premier sonnet, Parikia, qui lui donne cependant une tournure particulière. Plus, dit le sonnet, nous chaulons nos maisons à blanc et remplissons nos jardins de fleurs, plus nous avons le sentiment de cacher quelque chose que nous ne comprenons que lorsqu'au large, nous nous retournons et regardons en arrière. Mais le 
vent qui souffle des portes et fenêtres de ces maisons est si fort que nous devons tellement lutter pour rentrer que nous n'avons jamais le temps

de retenir la vue de nous-mêmes à distance suffisamment longtemps pour comprendre que nous sommes devenus cette distance ${ }^{21}$.

Le Moi, dont la maison est la métaphore - comme chez un autre poète danois, exilé (en Espagne dans les années 1950) comme Nordbrandt: Ole Sarvig -, n'est pas dépositaire de l'identité. Elle réside dans la distance établie par rapport au Moi. C'est seulement à distance que l'on voit clairement ce qui est trop près. La thérapie psychanalytique qui consiste à se rapprocher de plus en plus du Moi et de son subconscient, éloigne ; l'éloignement, paradoxalement, rapproche. On entend aussi dans ce sonnet comme un écho thématique lointain du récit de l'aiglon Klaus chez Henrik Pontoppidan (1857-1943). Ayant quitté la basse-cour du pasteur pour suivre un jeune aigle Klaus est pris de vertige au-dessus des montagnes sauvages et fait demi-tour. Mais le domestique du pasteur, ne reconnaissant pas Klaus, le prend pour ce qu'il est devenu, un aigle, et le tue. Le même destin rôde-t-il dans les derniers vers du poème de Nordbrandt dont le sens serait alors: nous nous tuons dans la lutte pour revenir. Absurde parce qu'inévitable. Le seul plaisir reste donc ce moment de prise de conscience avant le retour. Selon les mots d'Albert Camus au sujet de Sisyphe qui, pendant qu'il descend sa montagne, regarde les paysages : il faut imaginer Sisyphe heureux! En effet, nombre de " poèmes méditerranéens " de Nordbrandt reflètent ce plaisir sensuel que lui offre la Grèce qui, a-t-il dit, est tout simplement le plus grand défi lancé aux sens que je connaisse. Et quand les sens sont poussés à bout, l'intellect se purifie. À aucun autre endroit on ne voit comme dans la lumière de la Grèce ${ }^{22}$.

Plus tard, l'identité est multiple :

Chaque jour je suis un autre que le jour précédent

et pénètre davantage dans l'obscurité

[...]

Je suis à la fois Hélène et les Hellènes

les rameurs qui rentrent les proues sculptées dans l'aurore

et chaque rameur qui, enchaîné à sa rame,

rame avec le sentiment de ne jamais avancer.

On pense à Johannes V. Jensen à la gare de Memphis.

Le Moi de ces poèmes semble scindé en deux : une partie qui voit, observe ; et une autre qui sait d'avance ce qui arrivera et qui, par là, rend vain tout effort. La structure évoque celle du poème Arabesque, de J.-P. Jacobsen (1847-1885), qui commence par raconter comment une vague s'arrache de sa forme élémentaire pour se transformer en cygne. Mais après quelques battements d'aile, seulement, son envol est interrompu par une voix savante qui lui raconte, en un poème autonome, son destin inévitable qui est celui de se consumer dans l'angoisse - autre forme de la dialectique du désir «nordique ». On a l'impression que ce "poème dans le poème » draine littéralement l'énergie de la vague pour l'investir dans des images colorées à l'excès et structurées en arabesques, sorte de circuit fermé qui finit par consumer cette énergie jusqu'à ce qu'il n'en subsiste qu'un soupir :

Pourquoi la vie?

Pourquoi la mort?

Pourquoi vivre, quand il faut quand même mourir? 
Pourquoi lutter quand nous savons que le glaive

Sera même un jour arraché à nos mains ?23

Dans un autre poème de Glas, de caractère onirique, De tous les nombreux personnages, un seul se dégage de ces nombreuses facettes qui constituent le Moi. Il se tient calme et solitaire dans un paysage rempli de gens qui se combattent. Le Moi veut les approcher pour leur dire qu'ils ont été trahis, que ce sont eux-mêmes qu'ils combattent, mais le personnage lui en interdit l'accès :

[...] calme, les yeux à moitié fermés

avec des silhouettes ensanglantées qui tombent autour de lui

des silhouettes ensanglantées qui toutes auraient pu être moi

il m'observe, de plus en plus seul

au fur et à mesure que les rangs des vivants

se rétrécissent et ceux des morts se rallongent

- Jusqu'à ce que je vois mes traits se mêler aux siens,

figés, tordus, haineux et moqueurs,

et sens son aile de fer se refermer sur moi ${ }^{24}$.

Qui est-il cet être cruel et impassible voire insensible? L'esthète, le poète qui des souffrances des autres ne tire que le plaisir esthétique et dont l'étreinte est liée à la mort? Peut-on, pour rester dans la métaphore de Jacobsen, comparer le Moi de Nordbrandt à la vague et la personne aux ailes de fer à ce savoir préexistant de la vanité de toute chose qui draine l'énergie de la vague-Moi et fait de sa démarche une lutte sans espoir? Aurait-on affaire à la lutte de Jacob avec l'ange noir de l'esthétique, version Henrik Nordbrandt? À moins qu'il faille y voir un cauchemar de plus sur l'impuissance d'atteindre son but parce qu'on est soi-même celui qui l'empêche. La seule issue sera alors l'élimination pure et simple de ce Moi. Car

Seul un mort

peut doucement entrer en lui-même

au travers de ces murs blancs ${ }^{25}$.

On rencontre effectivement nombre de cadavres dans les poèmes de Nordbrandt, des cadavres que le Moi reconnaît comme étant les siens. Ainsi dans Lesbos:

Parcourir ce paysage est un privilège pour les rêveurs :

Se réveiller sous un olivier, tard l'après-midi

pour siffler une note de flûte, longue et mélancolique

que tu as appris par des ravins jamais franchis

sur un pipeau : Arriver à un village isolé dans les montagnes

par un chemin, sous de grands peupliers dont le bruissement

résonne comme celui de l'été à l'écoute de lui-même: Ouvrir une porte peinte en

bleu

qui mène dans un jardin frais où ils pleurent un cadavre

que tu reconnais comme étant le tien. [... ${ }^{26}$

On pourrait formuler le paradoxe ainsi : pour entrer en soi il faut sortir de soi. Le recueil Ode à la pieuvre est entièrement consacré à ce paradoxe accompagné tout au long par la figure de Yunus Emre (env. 1240-1320), un des grands mystiques de la poésie turque. C'est pourquoi Nordbrandt appelle ces poèmes «poèmes d'amour » - dans le sens des mystiques : 
Dans l'imagination créatrice, le sensible et le non-sensible se transforment. Le sensible est habité d'un mouvement ascendant, le non-sensible d'un mouvement descendant ; telle est la dialectique de la rencontre entre l'amour charnel et l'amour spirituel, qui engendre l'amour mystique, point de fusion des deux. Cet amour conduit à un état de « conscience suprême » ou d'" existence suprême » dans lequel les deux amants s'unissent en oubliant toute limite. Le mystique se sent libéré : il n'est rentré en lui-même que pour mieux s'échapper, il sort de lui-même et se soustrait aux limites naturelles de la sensation purement corporelle. [...] Le Moi y est conçu comme mouvement incessant vers l'Autre, et pour qu'il atteigne l'Autre, il faut se dépasser. Le Moi ne peut voyager vers la profondeur de sa propre existence que dans la mesure où il voyage vers l'existence profonde de l'Autre. Il ne trouve sa parfaite présence que dans l'Autre; le Moi est, paradoxalement, non-Moi. Vue sous cet angle, l'identité est comme l'amour : elle est création continue ${ }^{27}$.

On ne peut pas vraiment dire que Nordbrandt est devenu mystique. Mais tout comme Lao Tzu, quelques années auparavant, lui avait donné une interprétation positive aux thèmes du vide et de l'absence ${ }^{28}$, on peut dire qu'avec le mysticisme arabe, il a trouvé une poésie et une vision de l'existence qui donne à ses propres problèmes existentiels et identitaires une interprétation positive et pure dans ce sens qu'elle n'est liée à aucune institution confessionnelle :

Maintenant elle m'a rempli ô Djelaleddin

la mer de l'amour dont tu parles.

Sur trois plages, celle que j'appelle mienne

trouva mon corps, et j'étais elle, un et vide ${ }^{29}$.

L'amour c'est la présence de l'être aimé, concrètement. Mais c'est aussi un état d'âme, un mode de vie indépendant du temps et du lieu, quelque chose d'absolu qui ne suppose nullement la présence de l'aimée :

Parfois je suis si près de toi que je pleure

Parfois je suis si loin de toi que je ris.

Parfois le ciel printanier au-dessus des toits est si bleu

qu'il apparaît très clairement pourquoi l'amour est ainsi ${ }^{30}$.

Dieu - l'être aimé, le Tu du poème peut être les deux à la fois. L'Autre de toute façon. Mais où investir le désir quand il n'est pas là ? La réponse est: dans le poème, la langue :

C'est si terrible d'écrire sur toi

que je ne sais pas où placer mes mots

sans me brûler. D'un autre côté,

je n'ai nulle part ailleurs où aller quand tu n'es pas ici ${ }^{31}$.

Ce qui fait du poème, de la langue, un organe sensoriel de plus. Comme chez W.B. Yeats :

Caught in that sensual music all neglect

Monuments of unaging intellect ${ }^{32}$.

Henrik Nordbrandt :

Les distances brûlent nos corps comme de l'opium.

Jamais nous n'avons été si riches en lieux.

Nous retournons nuit après nuit avec nos cendres

- sans avoir jamais quitté

Le pays de feu vers lequel nous voyageons dans nos chansons ${ }^{33}$. 
Cette musique sensuelle reste pour toujours la marque de noblesse de la poésie de Henrik Nordbrandt qui avec les années s'emplit de plus en plus de l'Autre. Adonis remarque, au sujet du mystique, qu'il devient, dans la métaphore, objectivement Autre. Ce n'est donc plus lui qui énonce le sens et qui le traduit en image : c'est le sens lui-même qui énonce et écrit l'image. Ce n'est pas le mystique qui pense et qui écrit, il est pensé et il est écrit ${ }^{34}$. Dans Guds Hus (La Maison de Dieu), Henrik Nordbrandt fait une expérience similaire, celle de ne pas voir, mais d'être vu - non pas par soi-même, comme dans les poèmes schizophrènes, mais par un autre ordre dans lequel il est d'avance inscrit :

Moi, qui ne crois en rien,

je sais que cette eau est sacrée.

Celui qui la boit

se voit lui-même depuis les montagnes

qu'elle a baptisées ${ }^{35}$.

Il était précédemment question d'abstractions et d'un personnage qui empêchait le Moi d'approcher les gens qui se combattaient mutuellement. Vu d'ici, de l'expérience nommée Guds hus, force est de conclure que l'écriture poétique même faisait partie des abstractions (dans une interview de 1982, il compare, avec une métaphore empruntée à Gotfried Benn, la poétique de ses premiers poèmes à l'activité de faire des bulles de savon qui étaient belles un instant durant puis disparurent $\left.{ }^{36}\right)$. La nouvelle stratégie consiste à faire du thème départ/arrivée la forme même du poème par le biais du paradoxe :

Je n'oublie jamais les choses que j'oublie de coucher sur le papier.

C'est ainsi que je sépare le concret de l'abstrait ${ }^{37}$.

L'abstraction mortelle de l'écriture est contrecarrée, annulée ou «ligaturée » par des «falsifications » qui visent une réalité psychique concrète telle que le silence, l'oubli ou la conscience de la mort:

Sous un titre pareil que seule peut supporter

la ligne ultime

j'exécute encore une fois la falsification

qui, démasquée, éclaire pourtant suffisamment

l'instant qu'il faut

d'avance s'abstenir d'envahir

de sorte qu'un amandier, subitement fleuri,

portera néanmoins

le fruit que je croyais avoir mangé ${ }^{38}$.

En conclusion: pour les deux écrivains, même point de départ: le malaise au Danemark, dans le monde; même réponse: le voyage. Mais deux issues différentes. Johannes V. Jensen projette son désir devant lui qui transforme les lieux en son image. Il veut que la réponse lui vienne du dehors, du lieu, et doit par conséquent repartir à chaque fois avec le sentiment enrageant d'être frustré de son destin fulgurant ${ }^{39}$.

Henrik Nordbrandt en revanche se laisse pénétrer par les paysages et lieux étrangers. Ce sont eux qui formulent son désir et non l'inverse. Aussi sa poésie s'ouvre à de plus en plus de réalité, non-transformée - intérieure et extérieure, contemporaine et historique comme dans Arménia - donnant à sa poésie son chant particulier. 


\section{NOTES}

1. Johannes V. Jensen : Digte 1906, Gyldenlas spættebøger, 1966, p. 56. Les traductions de Johannes V. Jensen et de Henrik Nordbrandt sont de l'auteur du présent article.

2. Digte 1906, op. cit., p. 57.

3. Digte 1906, op. cit., p. 18.

4. Danois : sammenbidthed, litt. : « le fait de serrer les mâchoires ».

5. Johannes V. Jensen : Digte 1906, op. cit., p. 59.

6. Drømmebroer, 1998, p. 13.

7. Glas, 1976, p. 14.

8. Opbrud og ankomster, 1974, p. 53.

9. Opbrud og ankomster, op. cit., p. 65.

10. Opbrud og ankomster, op. cit., p. 45.

11. Grece, Glas, op. cit., p. 69.

12. Opbrud og ankomster, op. cit., p. 69.

13. Gunnar Ekelöf, Strountes, 1955.

14. Simi, Opbrug of ankomster, op. cit., ibid., p. 10.

15. Opbrud og ankomster, op. cit., p. 35.

16. Emmanuel Lévinas : Autrement qu'être ou au-delà de l'essence, 1978. Livre de Poche, 1996, p. 99.

17. Digte (1966), Miniaturer (1967), Istid (1977), Spøgelseslege (1979).

18. Syvsoverne (1969), Omgivelser (1972), Opbrud of ankomster (1974), Ode til bloeksprutten (1975), Glas (1976), Guds hus (1977).

19. Glas, op. cit., p. 35.

20. Glas, op. cit., p. 9.

21. Dans ses premiers recueils, Nordbrandt considérait l'écriture comme une sorte de thérapie. Voir interview 1982, in ; Erik Skyum-Nielsen : Modsprogets poetik, Arena, 1982, p. 114.

22. Breve fra en ottoman (Lettres d'un Ottoman). Essais, 1978, p. 138.

23. Glas, op. cit., p. 37.

24. J.-P. Jacobsen: Arabesque (dessin de Michel Ange représentant de profil une femme aux regards baissés et se trouvant au Palais des Offices). Trad. Frédéric Durand. In: F.J. Billeskov Jansen: Anthologie de la littérature danoise, Aubier Montaigne, Paris, 1964, p. 573.

25. Glas, op. cit., p. 59.

26. Naoussa ; Glas, op. cit., p. 29.

27. Opbrud of ankomster, op. cit., p. 29.

28. Adonis : Expérience et identité. Introduction à une nouvelle lecture du mysticisme arabe (1989). In : La prière et l'épée (Essais sur la culture arabe), Mercure de France 1993, pp. 244-245, 247.

29. Syvsoverrne, 1969.

30. Ode til blokksprutten, p. 35.

31. Ode til blœkksprutten, op. cit., p. 42.

32. Ode til blokksprutten, op. cit., p. 41.

33. W.B. Yeats : Sailing to Byzantium. The Penguin Poetry Library, W.B. Yeats, Selected poetry, Londres, 1991, p. 128.

34. Glas, op. cit., p. 32.

35. Adonis, op. cit., p. 240-241.

36. Guds hus, 1977, p. 31.

37. Erik Skyum-Nielsen, op. cit., p. 115.

38. Glemmesteder (Lieux d'oubli), 1991, p. 11.

39. Glemmesteder, op. cit., p. 59. 


\section{RÉSUMÉS}

Parti faire le tour du monde pour trouver son «destin fulgurant», Johannes V. Jensen fait à chaque fois l'expérience amère $d$ être en dehors. Parce que le lieu qui satisfera son désir doit avoir les mesures exactes de ce même désir, l'imagination s'ingénie à les transformer. Aussi, quand l'énergie a été consumée, ils apparaissent encore plus décevants qu'ils ne l'étaient. C'est ce que nous avons appelé la dialectique du désir. Pour sortir du dilemme, Johannes V. Jensen investit son désir dans le temps et non dans l'espace. Mais c'est la même chose qui se produit : le désir transforme le darwinisme, qui chez Jensen tient lieu de théorie anti-métaphysique, afin qu'il soit à sa mesure.

Henrik Nordbrandt fait l'expérience inverse. En se laissant pénétrer par les paysages étrangers, il arrive à extérioriser le désir, en ce sens que ce n'est pas le désir qui façonne les lieux et paysages à son image, mais inversement, les paysages qui formulent le désir, c'est-à-dire le rendent visible. Ainsi sa poésie se remplit de plus en plus de réalité concrète et vécue, opposée à l état actuel de la civilisation jugée de plus en plus abstraite.

Johannes V. Jensen, der sich auf Weltreise begibt, um sein «Schicksal » zu finden, macht jedes mal die bittere Erfahrung, außen vor zu sein. Da der Ort, der seine Sehnsucht stillen könnte, exakt die Maße eben dieser Sehnsucht haben müsste, bemüht sich die Einbildungskraft, diese zu verwandeln. Außerdem erscheinen die Orte, sobald die Energie aufgebraucht ist, noch enttäuschender dar sich als zuvor. Dies haben wir Dialektik der Sehnsucht genannt. Um diesem Dilemma zu entziehen, macht Johannes V. Jensen die Zeit. Aber es passiert das gleiche : die Sehnsucht verwandelt den Darwinismus, der bei Jensen die Position einer anti-metaphysischen Theorie einnimmt, so, dass er ihr angemessen sei.

Henrik Nordbrandt macht die entgegengesetzte Erfahrung. Indem er sich von den fremden Landschaften durchdringen lässt, gelingt es ihm, die Sehnsucht zu veräußerlichen, in dem Sinne, dass nicht die Sehnsucht die Orte und Landschaften nach ihrem eigenen Bild formt, d.h. sie verwandelt, sondern umgekehrt die Landschaften die Sehnsucht ausdrücken, d.h. sie sichtbar machen. Auf diese Weise füllt sich seine Dichtung mehr und mehr mit konkreter und erlebter Wirklichkeit, im Gegensatz zum aktuellen Zustand der Zivilisation, der als mehr und mehr abstrakt bewertet wird.

\section{AUTEUR}

\section{KARL EJBY POULSEN}

Université de Paris IV-Sorbonne 